\title{
Univ.-Prof. i.R. Dipl.-Ing. Dr. mont. Bruno Buchmayr
}

\author{
Gerhard Hackl, Willibald Mautner, Horst Cerjak, Arnold Letschnik und Martin Stockinger
}

Leoben, Österreich

Angenommen 19. Februar 2021; online publiziert 9. März 2021

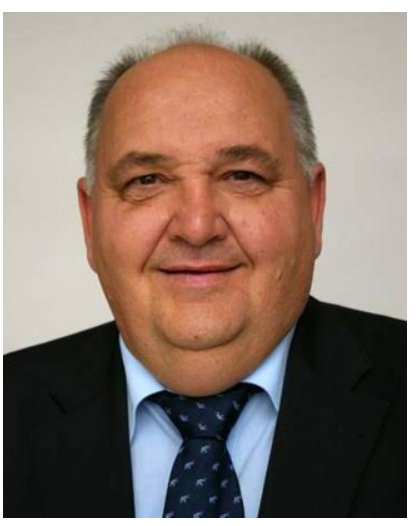

Wir verabschieden uns von einem großartigen Menschen, Werkstoffwissenschaftler und Visionär, der am 8. Februar 2021 im Alter von 66 Jahren nach kurzer schwerer Erkrankung verstorben ist.

Bruno Buchmayr wurde am 17. März 1954 in Leoben geboren. Nach seiner Matura an der HTBL in Kapfenberg und seinem Bundesheerdienst inskribierte er im WS 1974/75 für die Studienrichtung Werkstoffwissenschaften an der damals Montanistischen Hochschule in Leoben. Im Juni 1980 schloss er sein Studium ab. Schon während seines Studiums war Bruno Buchmayr als Studienassistent am Institut für angewandte Mathematik tätig. Nach dem Abschluss des Studiums war er von 1980 bis 1983 als Vertragsassistent am Institut für Metallkunde und Werkstoffprüfung angestellt. Im Dezember 1983 schloss er unter Prof. Hellmut Fischmeister seine Doktorarbeit mit dem Thema: „Analyse des Ermüdungs- und Kriechverhaltens der Nickelbasislegierung IN738LC im Einsatz für Turbinenschaufeln bei Gasturbinen" ab.

Im Oktober 1983 wechselte Bruno Buchmayr an die Technische Universität Graz an das Institut für Werkstoffkunde und Schweißtechnik. 1988-1989 erhielt er im Rahmen eines FWF-Erwin Schrödinger Stipendiums die Möglichkeit

\footnotetext{
G. Hackl (网)

Leoben, Österreich

Gerhard.hackl@ asmet.org
}

eines Gastaufenthalts an der McMaster University in Hamilton in Ontario, Canada. Im Jahr 1991 wurde inm die "venia legendi“ für Werkstoffkunde und Schweißtechnik an der Technischen Universität Graz erteilt. Im selben Jahr startete auch das von ihm geleitete sehr erfolgreiches CD-Labor für "Computermodellierung werkstoffkundlicher Vorgänge und Verarbeitungstechnologien", das bis 1998 lief. 1997 wurde er a.o. Univ.-Prof. am Institut für Werkstoffkunde, Schweißtechnik und Spanlose Formgebungsverfahren an der TU-Graz.

Nach seinem CD-Labor wirkte er entscheidend an der Entstehung von Kompetenzzentren wie dem Materials Center Leoben, in dem er seit 1999 als key-researcher tätig war, oder dem Kompetenzzentrum "Das Virtuelle Fahrzeug vif" an der TU-Graz mit.

Bruno Buchmayr gehörte zu den Pionieren in dem Bereich computerbasierende Modellierung von Werkstoffen bei der Produktion und im Einsatz. Das von ihm verfasste Buch „Computer in der Werkstoff- und Schweißtechnik: Anwendung von mathematischen Modellen" gehört heute zu den meist gelesenen Werken auf diesem Gebiet. 2002 erschien sein Lehrbuch „Werkstoff- und Produktionstechnik mit Mathcad-Modellierung und Simulation in Anwendungsbeispielen".

Mit 1. Februar 2003 wurde Bruno Buchmayr als Professor für Umformtechnik an die Montanuniversität berufen. Er setzte seinen wissenschaftlichen Schwerpunkt weiter fort und verknüpfte erfolgreich die Bereiche Simulation, Werkstoffverhalten und Konstruktion im Bereich der Umformtechnik. Bruno Buchmayer war stets bemüht, an Zukunftsthemen mitzuarbeiten. So brachte er den 3D-Druck für Metalle auf die Montanuniversität Leoben und bemühte sich, die Digitalisierung an der Montanuniversität zu verankern.

Bruno Buchmayr war mehr als 15 Jahre Mitglied des Senats der Christian Doppler Forschungsgesellschaft und Beirat im Bundesministerium bezüglich Förderschiene „Produktion der Zukunft" - und Wegbereiter mehrerer CD-Labors an der Montanuniversität Leoben. Bruno Buchmayr war aber auch im Vorstand der ASMET über 15 Jahre tätig und Mitbegründer des Fachausschusses Umformtechnik. Seine mehr als 240 Publikationen, davon auch einige 
in unserer Zeitschrift BHM, sowie insgesamt 28 von ihm betreute Doktoranden bestätigen seine wissenschaftliche Exzellenz und seinen Willen, dieses Wissen auch zu verbreiten. Dieses zeigt sich einerseits in der Vielzahl neuer zukunftsorientierter Lehrveranstaltungen, die in seiner Wirkungsphase an der Montanuniversität entstanden sind, aber andererseits auch in der Organisation mehrerer internationaler Tagungen und der regen Vereinstätigkeit, wie in der wissenschaftlichen Gesellschaft für Produktionstechnik, der er als Präsident von 2015 bis 2017 vorstand. Seine wissenschaftlichen Leistungen wurden auch durch eine Vielzahl von Preisen, wie dem Hans-Malzacher-Preis der EHÖ (ASMET), Prof. Masubuchi-Shinsho Corp. Award der American Soc. for Welding, Franz-Leitner-Preis der EHÖ (ASMET), ASM European Lecturer, Fellow ASM und dem Forschungspreis des Landes Steiermark 1998, anerkannt.

Neben den vielen herausragenden wissenschaftlichen Leistungen von Bruno Buchmayr hat er auch immer einen sehr engen Kontakt zur heimischen Industrie gepflegt. Das von ihm auf Basis physikalischer Grundlagen entwickelte Modell des thermo-mechanischen Walzens über Prozessmodellierungen wurde weltweit erstmalig zur Onlinesteuerung von Walzprozessen an der Breitbandstrasse der VA Linz und im Drahtwalzwerk Donawitz realisiert. Weitere Beispiele der konsequenten Anwendung von auf mikrostruktureller Basis fundierter Umsetzung von Modellbildung und Simulation sind seine Beiträge für das Rohrwalzwerk Kindberg, Böhler Schmiedetechnik und Böhler Mehrlinienwalzwerk. Damit hat Bruno Buchmayr wesentlich zu den großen Erfolgen, die die österreichische Stahlindustrie in den letzten Jahrzehnten erlebt hat, beigetragen.

Bruno Buchmayrs Engagement ging aber über die wissenschaftliche Tätigkeit auf universitärem Boden weit hinaus, und er war auch im öffentlichen Leben der Stadt Leoben ein wesentlicher Impulsgeber und Initiator. So geht auf ihn die Gründung und Betreuung der offenen Werkstätte für moderne Fertigung „FabLab Leoben“ zurück. Bruno Buchmayr war auch federführend bei der Durchführung von Ausstellungen in der Stadt Leoben engagiert. Die Ausstellungen zum Thema "Fertigungstechnik am Beispiel des Automobilbaues" und "Raumfahrt" sind hier zu nennen. Ein weiterer Schwerpunkt von Bruno Buchmayr war die Motivation junger Menschen für die Technik und insbesondere für montanistische Studienrichtungen. So organisierte er die Ausstellung „Material4Kids", um schon sehr früh mit der Motivation unseres Nachwuchses zu beginnen. Für die vielen Bemühungen um die Stadt Leoben wurde Bruno Buchmayr das goldene Ehrenzeichen der Stadt Leoben verliehen.

Für Bruno Buchmayr hatte die Familie einen besonderen Stellenwert in seinem Leben. Im April 1981 heiratete er Gabi, und sie konnten sich über die Geburt von ihren Kindern Birgit, Michi und Martin freuen, die in den Jahren 1982, 1983 und 1987 zur Welt kamen. Die Kinder waren sein Stolz, und er förderte stets deren unterschiedliche Begabung.

Seine Vielseitigkeit spiegelt sich auch in seiner Freizeit wider. Eine seiner Leidenschaft war die Malerei. Es machte ihm Spaß verschiedene Maltechniken zu erlernen, und er malte wunderschöne Bilder für seine Familie und seine Freunde.

Mit seinen vier Enkelkindern verbrachte er viel Zeit in seiner „Räuberhöhle“ (seinem Büro im Haus) mit Basteln, Malen und vielen anderen lustigen Aktivitäten.

Mit dem Schifahren war es Bruno möglich, Beruf und Freizeit zu verbinden. Mit großer Begeisterung und Engagement organisierte Bruno Buchmayr gemeinsam mit dem Lehrstuhl für Umformtechnik der Montanuniversität die Umformtechnischen Kolloquien auf der Planneralm und anschließend in Zauchensee. Diese Umformtechnischen Kolloquien zählen zu den bedeutendsten wissenschaftlichen Veranstaltungen in Österreich auf dem Gebiet der Umformtechnik.

Ein besonderes Faible hatte Bruno für die italienische Kultur, das italienische Essen und die italienische Sprache. So verbrachte er gemeinsam mit Gabi viele Urlaube in Italien, das letzte Mal im Herbst 2019 im Gebiet des südlichen Gardasees mit Radausflügen entlang des Flusses Mincio.

Allein aus der Aufzählung seiner Leistungen wird klar, wie erfolgreich und beeindruckend sein Wirken in den Bereichen Werkstoffkunde, Produktionstechnik und Öffentlichkeitsarbeit war. Die österreichische Forschungslandschaft und Industrie verlieren einen weit über die Grenzen hinaus anerkannten Forscher und Wissenschaftler.

Bruno Buchmayr war ein visionärer aber stets ruhiger, zurückhaltender, ja sogar bescheidener Mensch, der trotz seiner Erfolge nicht überheblich war und sehr diszipliniert und fachlich überaus fordernd Schritt für Schritt seinen Lebensweg ging. Er war überaus korrekt, aber auch hilfsbereit, tiefsinnig, gläubig und für viele ein sehr guter Freund und Mentor.

Er war aufgrund seines vielfältigen Wirkens immer ein Vorbild, und wir werden ihm stets ein ehrendes Andenken bewahren.

Unser aller Mitgefühl und unsere innigste Anteilnahme gelten seiner Frau Gabi, den drei Kindern Birgit, Michi und Martin mit Familien sowie allen, die einen wertvollen Menschen verloren.

Fiducit!

Gerhard Hackl

Willibald Mautner

Horst Cerjak

Arnold Letschnik

Martin Stockinger 